\title{
Interferência de ferro na dosagem de boro no solo com azometina-H em soluções extratoras ácidas ${ }^{(1)}$
}

\author{
Gilvan Barbosa Ferreira(2), Renildes Lúcio Ferreira Fontes ${ }^{(3)}$, Victor Hugo Alvarez Venegas ${ }^{(3)}$, \\ Maurício Paulo Ferreira Fontes ${ }^{(3)}$ e Juliana Maria Nogueira Pereira ${ }^{(2)}$
}

\begin{abstract}
Resumo - A busca de métodos que permitam a extração do boro para avaliação da fertilidade do solo para a recomendação de adubação é importante, devido aos baixos teores de boro e aos altos teores de Fe na maioria dos solos brasileiros. A extração com água quente, método-referência, é trabalhosa e apresenta dificuldades operacionais. Por outro lado, soluções de $\mathrm{CaCl}_{2} 5 \mathrm{mmol} / \mathrm{L} \mathrm{e} \mathrm{BaCl}_{2} \cdot 2 \mathrm{H}_{2} \mathrm{O} 5 \mathrm{mmol} / \mathrm{L}$ são usadas com sucesso. O objetivo deste trabalho foi testar a eficiência do ácido tioglicólico na eliminação da interferência do Fe na dosagem de B do solo com azometina-H, após extrações com soluções ácidas de Mehlich-1 e HCl (50 e 100 mmol/L). Dosagem com azometina-H, com e sem ácido tioglicólico, foram feitas para oito solos de Minas Gerais e dois solos do Mato Grosso do Sul. Foi utilizada como referência, a extração com $\mathrm{BaCl}_{2} .2 \mathrm{H}_{2} \mathrm{O} 5 \mathrm{mmol} / \mathrm{L}$, com aquecimento em forno de microondas e dosagem com azometina-H; a determinação de B com azometina-H em extratos originados de soluções ácidas sofre forte interferência do Fe. A adição do ácido tioglicólico não remove esta interferência. A coloração do complexo B-azometina-H fica menos estável com a adição do ácido tioglicólico na dosagem do boro.
\end{abstract}

Termos para indexação: ácido tioglicólico, solução, análise de solo, fertilidade do solo.

\section{Interference of iron on soil boron determination with azomethine-H in acid solutions}

\begin{abstract}
The search for methods to extract boron for evaluation of soil fertility to recommend fertilizer application is important due to the low levels of boron and high levels of iron in most Brazilian soils. Extraction with hot water, the standard method, is a hard work method with operational difficulties. On the other hand, solutions of $\mathrm{CaCl}_{2} 5 \mathrm{mmol} / \mathrm{L}$ and $\mathrm{BaCl}_{2} \cdot 2 \mathrm{H}_{2} \mathrm{O} 5 \mathrm{mmol} / \mathrm{L}$ have been used successfully. The objective of this work was to test the efficiency of the thioglycolic acid as a remover of $\mathrm{Fe}$ interference in the dosage of B with azomethine- $\mathrm{H}$, after extraction from soil with acid solutions (Mehlich-1, 50 and $100 \mathrm{mmol} / \mathrm{L} \mathrm{HCl}$ ). Dosage with azomethine-H, with and without thioglycolic acid, in soil extracts from eight Minas Gerais State soils and two Mato Grosso do Sul State soils was performed. The reference method was the extraction with $5 \mathrm{mmol} / \mathrm{L} \mathrm{BaCl}_{2} \cdot 2 \mathrm{H}_{2} \mathrm{O}$ in the microwave oven and azomethine- $\mathrm{H}$ dosage. The determination of $\mathrm{B}$ with azomethine- $\mathrm{H}$ in extracts originated from acid extractions undergoes strong interference of Fe. The addition of thioglycolic acid does not remove this interference. The color of the B-azomethine-H complex becomes less stable as thioglycolic acid is added.
\end{abstract}

Index terms: thioglycolic acid, solutions, soil analysis, soil fertility.

\footnotetext{
(1) Aceito para publicação em 14 de janeiro de 2002.

Extraído da dissertação de mestrado do primeiro autor apresentada à Universidade Federal de Viçosa (UFV), Departamento de Solos, Viçosa, MG.

(2) UFV, Dep. de Solos, Av. P. H. Rolfs, s/no , CEP 36571-000 Viçosa, MG. E-mail: gilvan@solos.ufv.br, juliana@ solos.ufv.br

(3) UFV, Dep. de Solos. Bolsista do CNPq. E-mail: renildes@solos.ufv.br, vhav@mail.ufv.br, mpfontes@mail.ufv.br
}

\section{Introdução}

A dosagem de B com azometina-H tem grande aceitação por ser menos trabalhosa, não exigir o uso de ácidos concentrados, ser mais precisa e, principalmente, não sofrer interferência da maioria dos íons presentes nas concentrações usuais em extratos de 
solos (Parker \& Gardner, 1981; Jeffrey \& McCallum, 1988). Apesar dessas vantagens, o método é suscetível à presença de matéria orgânica dissolvida ou em suspensão (Gupta, 1979a), e à presença de altos teores de $\mathrm{Fe}$ extraídos por ácidos fortes diluídos (Zarcinas, 1995).

A faixa entre os teores de deficiência e toxidez de B no solo é estreita (Sims \& Johnson, 1991), fazendo-se necessárias a padronização e simplificação das técnicas analíticas de modo que elas sejam sensíveis, confiáveis e livres das interferências para sua dosagem, especialmente em solos deficientes neste micronutriente.

Na determinação do B do solo, é comprovada a eficiência do método da água quente proposto por Berger \& Truog (1939) e considerado padrão. Este método, entretanto, é trabalhoso, oferece dificuldade no controle preciso de temperatura nos procedimentos de aquecimento e resfriamento da suspensão solo-extrator, é restritivo em relação ao número de amostras analisadas por série, requer grande trabalho para limpeza do aparato de refluxo e liberação de espaço para a execução de novas análises, e é oneroso, devido à exigência de vidraria isenta em boro. Nos solos ácidos, a extração com água quente deixa a desejar quanto à determinação do B disponível para as plantas, do ponto de vista da definição conceitual de B disponível (Offiah \& Axley, 1993).

A extração de $\mathrm{B}$ do solo com soluções de $\mathrm{BaCl}_{2}$ ou $\mathrm{CaCl}_{2}$, em sacos de plástico aquecidos em forno de microondas, tem sido usada com sucesso (Abreu et al., 1994; Ferreira et al., 2001). Ferreira et al. (2001) confirmaram a necessidade de adição do carvão ativado para essa extração como recomendado por Gupta (1979b). O BaCl $2.2 \mathrm{H}_{2} \mathrm{O}$ flocula os colóides formados durante o aquecimento (Wear, 1965). A extração de B com solução contendo $1,25 \mathrm{~g} / \mathrm{L}$ de $\mathrm{BaCl}_{2} \cdot 2 \mathrm{H}_{2} \mathrm{O}$ foi testada e recomendada para rotina de determinação de $\mathrm{B}$ disponível em solos (Abreu et al., 1994).

A extração de B com ácidos fortes diluídos, apesar de ser considerada inferior à extração com água quente (Cox \& Kamprath, 1972), tem sido usada com freqüência na avaliação do teor de B disponível no solo. Solução de HCl 50 mmol/L (Ponnamperuma et al., 1981; Renan \& Gupta, 1991), Mehlich-1 (Ribeiro \& Tucunango Sarabia, 1984; Bataglia \& Raij,
1990), HCl 50 mmol/Le HCl 100 mmol/L, e Mehlich-1 (Lima Filho, 1991) foram usadas como extratores do B disponível no solo. O uso de soluções ácidas é justificado pelos baixos custos da extração, incluindo o instrumental, e pela simplicidade de controle do tempo em comparação à extração com água quente ou suas adaptações. A extração com soluções ácidas torna-se especialmente importante para países com poucos recursos econômicos (Zarcinas, 1995).

Em solos ácidos, os métodos de extração de B com ácidos fortes diluídos podem extrair quantidades de Fe que interferem na dosagem de B com azometina-H. Em solos com teores baixos de boro $\left(<0,50 \mathrm{mg} / \mathrm{dm}^{3}\right)$ a interferência de Fe pode indicar através da análise química que esses solos tem B suficiente para as plantas (Zarcinas, 1995). Mesmo os métodos mais precisos de dosagem, como os que utilizam o ICP-AES, não conseguem eliminar a interferência do ferro. Jeffrey \& McCallum (1988) rejeitaram a linha de emissão mais sensível ao B $(\lambda=249,8 \mathrm{~nm})$ por haver sobreposição de espectro com o ferro. Esses autores, em extratos obtidos com $\mathrm{CaCl}_{2} 10 \mathrm{mmol} / \mathrm{L}$, em soluções contendo até $20 \mathrm{mg} / \mathrm{L}$ de $\mathrm{Fe}$, obtiveram limites de detecção de $0,01 \mathrm{mg} / \mathrm{L}$ para boro.

O objetivo deste trabalho foi testar a eficiência do ácido tioglicólico na eliminação da interferência de Fe na dosagem de B do solo com azometina-H após extrações com soluções ácidas de Mehlich-1 e HCl (50 e $100 \mathrm{mmol} / \mathrm{L}$ ).

\section{Material e Métodos}

Amostras de terra (TFSA), coletadas nas camadas superficiais $(0-20 \mathrm{~cm})$ de oito solos de Minas Gerais e dois do Mato Grosso do Sul, foram analisadas para determinação das características químicas e físicas (Tabela 1) e, em três repetições, submetidas à extração do $\mathrm{B}$ disponível, pelas soluções de Mehlich-1 (Bataglia \& Raij, 1990), HCl $50 \mathrm{mmol} / \mathrm{L}$ e HCl $100 \mathrm{mmol} / \mathrm{L}$ (Renan \& Gupta, 1991).

Os extratos foram filtrados em papel-filtro lento, usando-se dois papéis para cada filtração. Os extratos das três extrações de alguns solos foram misturados, por não ter sido obtido, em cada extração, volume suficiente para a dosagem programada.

Para fins de comparação, realizou-se a extração do B com $\mathrm{BaCl}_{2} 5 \mathrm{mmol} / \mathrm{L}$ (Abreu et al., 1994).

As dosagens com azometina-H, sem o ácido tioglicólico (SAT) (Parker \& Gardner, 1981) e com o ácido tioglicólico 


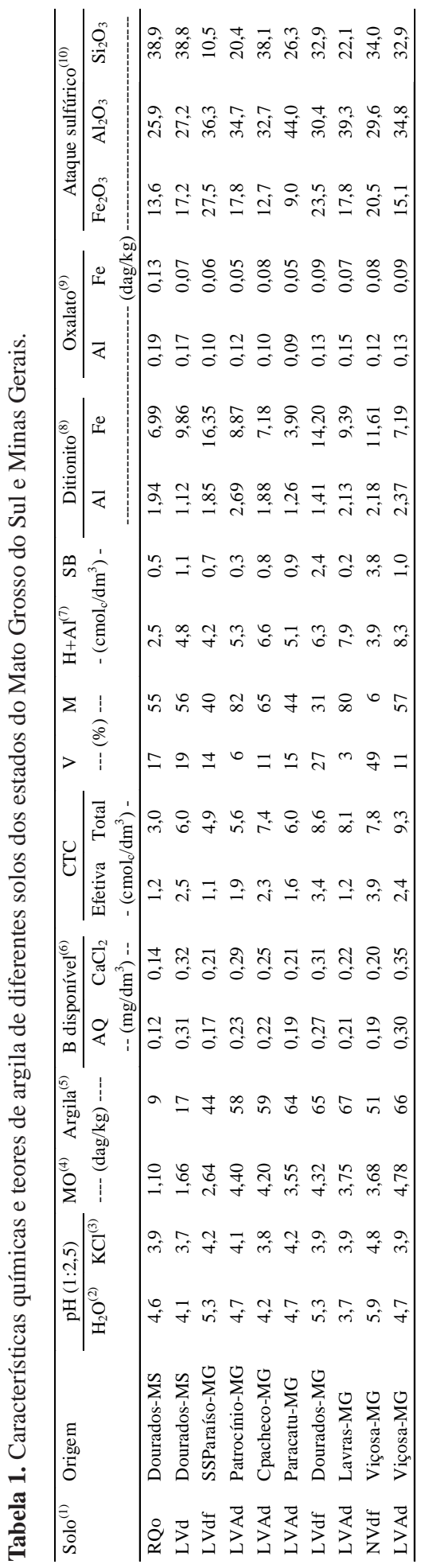

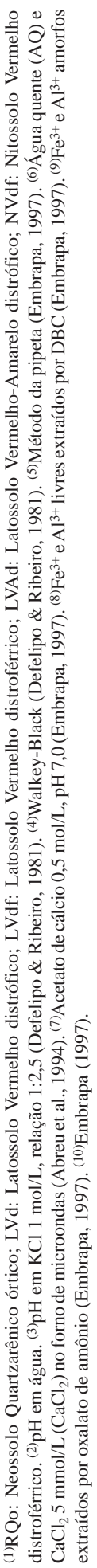

(CAT) (Zarcinas, 1995), foram realizadas logo após a filtragem.

Para cada extrator utilizou-se o delineamento em blocos ao acaso, num esquema fatorial completo 10x2 (10 solos e presença ou não do ácido tioglicólico) com três repetições. A presença do ácido tioglicólico objetivou testar a eliminação da interferência do ferro. As extrações foram realizadas sempre na presença do carvão ativado.

A interferência de Fe foi avaliada a partir de uma curvapadrão ajustada com soluções de Fe variando de 0 a $100 \mathrm{mg} / \mathrm{L}$ (solução padrão com 1.000 mg/L de Fe, Merck). Preparou-se, também, uma curva-padrão com soluções de B variando de 0 a $1 \mathrm{mg} / \mathrm{L}$. A seguir, realizou-se a determinação de boro. As soluções da curva-padrão para Fe receberam os mesmos reagentes, nas mesmas proporções, que foram adicionados às soluções usadas no preparo da curva-padrão para B, exceto o boro. Foram lidas, na seqüência, as soluções utilizadas no preparo da curva-padrão para B e as soluções utilizadas no preparo da curva-padrão para $\mathrm{Fe}$, sem o boro. Assim, determinou-se a interferência do $\mathrm{Fe}(\mathrm{IntFe})$ nas soluções onde foram feitas as determinações de B pelo método da azometina-H adaptado por Zarcinas (1995). A IntFe medida desta forma pressupõe que o teor de $\mathrm{B}$ detectado devido à interferência do Fe na dosagem com azometina-H é aditiva ao teor deste nutriente dosado no extrato.

Fazendo-se regressão entre os valores da IntFe obtidos e os teores de Fe determinados nas soluções utilizadas para preparo da curva-padrão de Fe, ajustou-se uma curva que permitiu determinar a IntFe nos extratos, para cada extrator, com e sem adição de ácido tioglicólico, para cada tipo de solo.

Os teores de B determinados (Parker \& Gardner, 1981) nas soluções padrões de Fe (0, 20, 40, 60, 80 e 100 mg/L) sem a presença do $\mathrm{B}$ e da azometina- $\mathrm{H}$ foram comparados com os teores de B obtidos nas soluções de sua curvapadrão (dosagem com azometina-H) para estimar a quantidade B, que, apesar de não estar presente, é contabilizado como se estivesse nos resultados analíticos de extratos ácidos de solo contendo ferro. Esse B 'aparente' é apresentado como interferência de ferro.

Tanto os dados dos teores de B disponível, determinados com adição (CAT) e sem adição (SAT) do ácido tioglicólico, como os dados referentes às IntFe, foram submetidos à análise de variância, para cada extrator ácido.

Foram feitos estudos de correlações entre teores de B extraídos com o método referência e os teores extraídos com os extratores ácidos, com e sem ácido tioglicólico. Os extratores foram correlacionados entre si pelo teste de correlação de Pearson. 


\section{Resultados e Discussão}

Os teores de B dosados com azometina-H, com ou sem a adição do ácido tioglicólico, não diferiram entre si em relação aos métodos de extração, exceto aos teores extraídos com $\mathrm{HCl} 100 \mathrm{mmol} / \mathrm{L}$ que foram inferiores quando determinados com a adição do ácido tioglicólico (Tabela 2). É possível que esta diferença se deva à adição do ácido tioglicólico, que proporcionou a supressão de alguma interferência de Fe na dosagem do B com azometina-H.

Os teores de Fe no solo foram crescentes (média de todos os solos), variando com o método de extração utilizado, na seguinte ordem: $\mathrm{HCl} 50 \mathrm{mmol} / \mathrm{L}$ $\left(17,92 \mathrm{mg} / \mathrm{dm}^{3}\right)<$ Mehlich-1 $\left(27,00 \mathrm{mg} / \mathrm{dm}^{3}\right)<$ $\mathrm{HCl} 100 \mathrm{mmol} / \mathrm{L}\left(62,35 \mathrm{mg} / \mathrm{dm}^{3}\right)$ (Tabela 3).

Os valores das interferências de Fe na dosagem de B com azometina-H, sem o ácido tioglicólico (IntFeSAT) e com o ácido tioglicólico (IntFeCAT), tiveram mesma variação em relação aos teores de $\mathrm{Fe}$ no solo (Tabela 3 ). Os valores de IntFeSAT e IntFeCAT mostram que o Fe presente nos extratos pode interferir absorvendo no comprimento de onda $420 \mathrm{~nm}$ e que o ácido tioglicólico pode reduzir essa interferência. Entretanto, os valores de interferência estão superestimados, o que pode ser decorrente dos métodos analíticos utilizados ou da redução da interferência devido à presença de $\mathrm{Na}_{2}$ EDTA na solução-tampão. De fato, os teores de B (Tabela 2) são, em diversas vezes, menores do que os valores das interferências de Fe estimadas (Tabela 3), e não diferiram significativamente entre os extratores $\mathrm{HCl} 50 \mathrm{mmol} / \mathrm{L}$ e Mehlich-1, quando analisados sem e com a adição do ácido tioglicólico (Tabela 2). Os métodos de análise não proporcionaram eficiência na avaliação quantitativa da interferência do $\mathrm{Fe}$ na determinação de $\mathrm{B}$ em extratos de solos obtidos com soluções ácidas. A dosagem de B por espectrometria de emissão atômica acoplada a plasma induzido (ICP-AES) representa uma alternativa de determinação e poderá ser útil para quantificar essa interferência. A vantagem de utilização de métodos colorimétricos são os custos menores das análises, o que, nas condições brasileiras, é de grande importância.

Uma séria dificuldade na dosagem do B disponível com ácido tioglicólico foi a falta de estabilidade na cor do complexo formado. Após a adição da azometina-H, os valores das leituras de absorvância em $420 \mathrm{~nm}$ (Zarcinas, 1995) permaneciam sempre instáveis. Constatou-se diminuição dos valores de absorvância, de forma rápida e proporcional em relação às concentrações de $\mathrm{B}$ nos pontos da curvapadrão, sendo que as leituras realizadas 80 minutos

Tabela 2. Teores de B determinados com azometina-H, sem adição (SAT) e com adição (CAT) de ácido tioglicólico, após extrações com diferentes extratores, em solos de Minas Gerais e Mato Grosso do Sul. Médias de três repetições.

\begin{tabular}{|c|c|c|c|c|c|c|c|}
\hline \multirow[t]{2}{*}{ Solo $^{(1)}$} & \multicolumn{2}{|c|}{ Mehlich-1 } & \multicolumn{2}{|c|}{$\mathrm{HCl} 50 \mathrm{mmol} / \mathrm{L}$} & \multicolumn{2}{|c|}{$\mathrm{HCl} 100 \mathrm{mmol} / \mathrm{L}$} & \multirow{2}{*}{$\frac{\mathrm{BaCl}_{2} \cdot 2 \mathrm{H}_{2} \mathrm{O}(1,25 \mathrm{~g} / \mathrm{L})}{\mathrm{SAT}}$} \\
\hline & SAT & CAT & SAT & $\begin{array}{l}\text { CAT } \\
-(\mathrm{m}\end{array}$ & $\begin{array}{c}\text { SAT } \\
3\end{array}$ & CAT & \\
\hline RQo & 0,26 & 0,28 & 0,17 & 0,20 & 0,37 & 0,33 & 0,13 \\
\hline LVd & 0,30 & 0,26 & 0,17 & 0,13 & 0,41 & 0,35 & 0,31 \\
\hline LVdf & 0,16 & 0,15 & 0,13 & 0,11 & 0,39 & 0,20 & 0,19 \\
\hline LVAd & 0,26 & 0,27 & 0,23 & 0,20 & 0,56 & 0,35 & 0,28 \\
\hline LVAd & 0,33 & 0,43 & 0,26 & 0,23 & 0,66 & 0,52 & 0,26 \\
\hline LVAd & 0,20 & 0,22 & 0,20 & 0,18 & 0,51 & 0,47 & 0,20 \\
\hline LVdf & 0,17 & 0,15 & 0,16 & 0,13 & 0,48 & 0,19 & 0,31 \\
\hline LVAd & 0,17 & 0,20 & 0,20 & 0,16 & 0,52 & 0,32 & 0,21 \\
\hline NVdf & 0,20 & 0,19 & 0,18 & 0,18 & 0,37 & 0,23 & 0,21 \\
\hline LVAd & 0,30 & 0,40 & 0,27 & 0,29 & 0,78 & 0,56 & 0,35 \\
\hline $\operatorname{Média}^{(2)}$ & $0,23 \mathrm{~A}$ & $0,25 \mathrm{~A}$ & $0,20 \mathrm{~A}$ & $0,18 \mathrm{~A}$ & $0,51 \mathrm{~A}$ & $0,35 \mathrm{~B}$ & 0,24 \\
\hline $\mathrm{CV}(\%)^{(3)}$ & $17,24 * *$ & 13,63 & 15,92 & 19,40 & 6,58 & 13,13 & 6,88 \\
\hline
\end{tabular}

(1)RQo: Neossolo Quartzarênico órtico; LVd: Latossolo Vermelho distrófico; LVdf: Latossolo Vermelho distroférrico; LVAd: Latossolo VermelhoAmarelo distrófico; NVdf: Nitossolo Vermelho distroférrico. ${ }^{(2)}$ Médias seguidas da mesma letra não diferem entre si pelo teste F a $5 \%$ de probabilidade. ${ }^{(3)} \mathrm{O}$ coeficiente de variação $(\mathrm{CV})$ se refere à média dos desvios-padrão por solo dividida pela média dos valores de todos os solos. 
Tabela 3. Teores de Fe (média \pm desvio-padrão) em extratos obtidos com Mehlich-1 e HCl e interferência do Fe na dosagem do B com azometina-H, sem adição (IntFeSAT) e com adição de ácido tioglicólico (IntFeCAT). Médias de três repetições.

\begin{tabular}{|c|c|c|c|}
\hline \multirow[t]{2}{*}{ Solo $^{(1)}$} & $\mathrm{Fe}$ & IntFeSAT & IntFeCAT \\
\hline & \multicolumn{3}{|c|}{ - } \\
\hline & \multicolumn{3}{|c|}{ Mehlich-1 } \\
\hline RQo & $53,29 \pm 6,18$ & $0,32 \pm 0,03$ & $0,22 \pm 0,02$ \\
\hline LVd & $27,65 \pm 2,51$ & $0,18 \pm 0,02$ & $0,15 \pm 0,01$ \\
\hline LVdf & $12,87 \pm 1,89$ & $0,09 \pm 0,01$ & $0,10 \pm 0,01$ \\
\hline LVAd & $20,98 \pm 1,18$ & $0,14 \pm 0,01$ & $0,13 \pm 0,00$ \\
\hline LVAd & $44,96 \pm 2,89$ & $0,28 \pm 0,02$ & $0,20 \pm 0,01$ \\
\hline LVAd & $36,46 \pm 1,76$ & $0,23 \pm 0,01$ & $0,17 \pm 0,00$ \\
\hline LVdf & $10,68 \pm 1,01$ & $0,07 \pm 0,01$ & $0,10 \pm 0,00$ \\
\hline LVAd & $22,78 \pm 2,35$ & $0,15 \pm 0,01$ & $0,13 \pm 0,01$ \\
\hline NVdf & $4,79 \pm 0,16$ & $0,04 \pm 0,00$ & $0,08 \pm 0,00$ \\
\hline LVAd & $35,53 \pm 1,45$ & $0,23 \pm 0,01$ & $0,17 \pm 0,00$ \\
\hline Média & $27,00 \pm 2,64$ & $0,17 \pm 0,01$ & $0,14 \pm 0,01$ \\
\hline \multirow[t]{2}{*}{$\mathrm{CV}(\%)^{(2)}$} & $9,78 * *$ & 7,98 & 4,86 \\
\hline & \multicolumn{3}{|c|}{$\mathrm{HCl} 50 \mathrm{mmol} / \mathrm{L}$} \\
\hline RQo & $30,58 \pm 2,86$ & $0,24 \pm 0,02$ & $0,08 \pm 0,01$ \\
\hline LVd & $17,14 \pm 0,56$ & $0,15 \pm 0,00$ & $0,05 \pm 0,00$ \\
\hline LVdf & $8,37 \pm 1,20$ & $0,09 \pm 0,01$ & $0,03 \pm 0,00$ \\
\hline LVAd & $15,98 \pm 1,36$ & $0,14 \pm 0,01$ & $0,05 \pm 0,00$ \\
\hline LVAd & $18,37 \pm 0,34$ & $0,30 \pm 0,00$ & $0,11 \pm 0,00$ \\
\hline LVAd & $24,40 \pm 2,27$ & $0,20 \pm 0,02$ & $0,07 \pm 0,01$ \\
\hline LVdf & $4,85 \pm 0,46$ & $0,07 \pm 0,00$ & $0,02 \pm 0,00$ \\
\hline LVAd & $14,47 \pm 0,46$ & $0,13 \pm 0,00$ & $0,04 \pm 0,00$ \\
\hline NVdf & $2,10 \pm 0,60$ & $0,05 \pm 0,00$ & $0,01 \pm 0,00$ \\
\hline LVAd & $22,90 \pm 3,72$ & $0,19 \pm 0,03$ & $0,06 \pm 0,01$ \\
\hline Média & $17,92 \pm 1,78$ & $0,16 \pm 0,01$ & $0,06 \pm 0,00$ \\
\hline \multirow[t]{2}{*}{$\mathrm{CV}(\%)^{(2)}$} & 9,94 & 7,84 & 9,02 \\
\hline & \multicolumn{3}{|c|}{$\mathrm{HCl} 100 \mathrm{mmol} / \mathrm{L}$} \\
\hline RQo & $70,90 \pm 2,59$ & $0,40 \pm 0,01$ & $0,22 \pm 0,01$ \\
\hline LVd & $58,02 \pm 2,33$ & $0,35 \pm 0,01$ & $0,19 \pm 0,01$ \\
\hline LVdf & $40,63 \pm 2,15$ & $0,26 \pm 0,01$ & $0,14 \pm 0,01$ \\
\hline LVAd & $51,38 \pm 7,40$ & $0,31 \pm 0,04$ & $0,17 \pm 0,02$ \\
\hline LVAd & $101,14 \pm 8,99$ & $0,52 \pm 0,03$ & $0,29 \pm 0,02$ \\
\hline LVAd & $80,19 \pm 1,28$ & $0,44 \pm 0,01$ & $0,24 \pm 0,00$ \\
\hline LVdf & $39,69 \pm 2,22$ & $0,25 \pm 0,01$ & $0,14 \pm 0,01$ \\
\hline LVAd & $60,16 \pm 1,67$ & $0,36 \pm 0,01$ & $0,19 \pm 0,00$ \\
\hline NVdf & $23,34 \pm 0,33$ & $0,16 \pm 0,00$ & $0,08 \pm 0,00$ \\
\hline LVAd & $97,82 \pm 2,25$ & $0,51 \pm 0,01$ & $0,29 \pm 0,00$ \\
\hline Média & $62,35 \pm 4,09$ & $0,36 \pm 0,02$ & $0,19 \pm 0,01$ \\
\hline $\mathrm{CV}(\%)^{(2)}$ & 6,56 & 4,74 & 5,07 \\
\hline \multicolumn{4}{|c|}{$\begin{array}{l}\text { (1)RQo: Neossolo Quartzarênico órtico; LVd: Latossolo Vermelho } \\
\text { distrófico; LVdf: Latossolo Vermelho distroférrico; LVAd: Latossolo Ver- } \\
\text { melho-Amarelo distrófico; NVdf: Nitossolo Vermelho distroférrico. }{ }^{\left({ }^{2}\right)} \\
\mathrm{CV}(\%) \text { se refere à média dos desvios-padrão por solo dividida pela média } \\
\text { dos valores de todos os solos. }\end{array}$} \\
\hline
\end{tabular}

após a leitura no ponto zero da curva eram, freqüentemente, mais baixas do que a leitura realizada no ponto zero da curva. Para superar essa dificuldade, todas as amostras foram lidas no intervalo entre 60 e 80 minutos após a adição da azometina-H.

A falta de estabilidade da cor deve estar ligada à alta concentração do ácido tioglicólico (200 mL/L) usada na dosagem. A maior leitura de absorvância no ponto da curva referente a $1 \mathrm{mg} / \mathrm{L}$ de B dificilmente ultrapassou o valor de 0,225 . Por outro lado, os valores de absorvância nesse mesmo ponto, sem adição do ácido tioglicólico, comumente se situaram próximos de 0,680 . Houve, portanto, uma alta perda de sensibilidade do método de dosagem quando o ácido tioglicólico era adicionado. Zarcinas (1995), quando propôs o método, chamou a atenção de que poderia haver perda de até $40 \%$ na sensibilidade em comparação ao método sem adição do ácido tioglicólico, entretanto, a diminuição da estabilidade da cor do complexo B-azometina-H não foi mencionada.

Interferências de $\mathrm{Al}, \mathrm{Cu}$ e Fe na dosagem de B com azometina-H em extratos de plantas são citadas por Gupta (1979a), o que sugere a possibilidade de interferências de Fe e Al na determinação de B disponível com azometina-H em solos ácidos.

Bingham (1982) recomenda que o $\mathrm{pH}$ da solução de leitura deve permanecer entre 4,9 e 5,1 para que a cor do complexo B-azometina-H se desenvolva satisfatoriamente. No presente trabalho, o pH da solução de leitura dos extratos obtidos com $\mathrm{BaCl}_{2}$ sempre alcançava $\mathrm{pH}$ 4,9 enquanto para os extratos obtidos com soluções ácidas o $\mathrm{pH}$ da solução de leitura, com ácido tioglicólico, variava de 4,5 a 4,7, e sem o ácido tioglicólico, de 4,3 a 4,5. Os valores de pH das soluções de leitura dos extratos variou conforme a acidez dos extratores: $\mathrm{HCl} 100 \mathrm{mmol} / \mathrm{L}<$ Mehlich-1 < $\mathrm{HCl} 50 \mathrm{mmol} / \mathrm{L}$. Entretanto, houve perda de estabilidade na cor do complexo B-azometina-H também nos extratos obtidos com $\mathrm{BaCl}_{2} 5 \mathrm{mmol} / \mathrm{L}$ quando a dosagem foi feita com azometina-H mais ácido tioglicólico, o que indica que a perda de estabilidade de cor está relacionada, principalmente, com a presença do ácido tioglicólico.

No presente trabalho o $\mathrm{HCl} 50 \mathrm{mmol} / \mathrm{L}$ foi o que proporcionou menores valores de interferência de Fe (Tabela 3), e os teores de B disponível com esse 
extrator também não se diferenciaram quando foram dosados com e sem a adição do ácido tioglicólico (Tabela 2), observando-se perda de sensibilidade e baixa estabilidade de cor na dosagem com azometinaH, com o ácido tioglicólico. Considerando-se aceitável a interferência do $\mathrm{Fe}$ associada ao método de dosagem com azometina-H (sem a adição do ácido tioglicólico), sua utilização é preferível em face do menor número de etapas do método, a não-utilização do ácido, que, além de tóxico, tem extremo mau cheiro, e a garantia de proporcionar maior estabilidade na cor e maior reprodutibilidade dos resultados.

Bataglia \& Raij (1990) constataram que os teores de B extraídos com $\mathrm{HCl} 100 \mathrm{mmol} / \mathrm{L}$ eram inconsistentes em virtude da forte coloração dos extratos, enquanto o Mehlich-1, ao contrário da água quente, foi ineficiente em discriminar o efeito da calagem e o efeito residual da adição do B ao solo.

A extração do B disponível com $\mathrm{HCl} 50 \mathrm{mmol} / \mathrm{L}$ foi eficiente em separar, com base nos teores de B, os solos tóxicos dos não-tóxicos em relação ao arroz inundado (Ponnamperuma et al., 1981). Nesse mesmo trabalho, constatou-se melhor correlação $\left(\mathrm{r}=0,91^{* *}\right)$ entre o teor de B disponível extraído com $\mathrm{HCl} 50 \mathrm{mmol} / \mathrm{L}$ e o conteúdo de B na planta, quando comparado aos resultados obtidos com a extração com água quente $(\mathrm{r}=0,84 * *)$. No entanto, esses autores encontraram menores teores de B na extração com $\mathrm{HCl}$ do que na extração com água quente, o que sugere uma limitação do $\mathrm{HCl}$ na previsão de deficiências de B nos solos ácidos quando a dosagem é feita com azometina-H. Vanderley (1984) constatou, em três solos do Município de Lavras, MG, que os teores de B extraídos com $\mathrm{HCl} 50 \mathrm{mmol} / \mathrm{L}$ tiveram boa correlação com os conteúdos de B nas plantas de girassol $\left(\mathrm{r}=0,94^{* *}\right)$ e com os teores de B determinados após extração com água quente $(\mathrm{r}=0,93 * *)$, como também menor extração de $\mathrm{B}$ com o $\mathrm{HCl}$ do que com a água quente. Deve-se mencionar que esses resultados se referem a extrações em solos que receberam adição de doses crescentes de boro.

Os extratores ácidos apresentaram correlações significativas entre si (Tabela 4) devido à ação dos extratores que deslocam o $\mathrm{B}(\mathrm{OH})_{3}{ }^{0}$ adsorvido aos minerais cristalinos do solo e à matéria orgânica $\mathrm{e}$ dissolvem a fração mais amorfa e recém-precipitada dos óxidos de ferro e alumínio do solo (Shuman, 1991;
Hou et al., 1996). Não houve correlação entre o B disponível determinado após extração com soluções ácidas e o $\mathrm{B}$ disponível determinado com $\mathrm{BaCl}_{2}$ $5 \mathrm{mmol} / \mathrm{L}$, com ou sem a adição do ácido tioglicólico, com exceção do $\mathrm{HCl} 100$ mmol/L com adição do ácido tioglicólico (Tabela 5). Como esses resultados ocorreram em tratamentos onde não se adicionou B ao solo, é reforçada a preocupação de Raij \& Bataglia (1991), com experimentos onde se faz a adição de doses crescentes de B ao solo para a seleção de extratores a partir de correlações com o conteúdo de B na planta. Pois, nesses experimentos, obtêm-se resultados próximos quando os extratores são comparados entre si, o que mascara a real eficiência do

Tabela 4. Correlações entre os métodos de extração de B com Mehlich-1 e HCl utilizando-se azometina-H para dosagem.

\begin{tabular}{|c|c|}
\hline Variáveis $^{(1)}$ & Coeficiente de correlação (r) \\
\hline $\mathrm{M}_{1}$ com $\mathrm{HCl}_{50}$ & $0,6698^{*}$ \\
\hline $\mathrm{M}_{1} \operatorname{com} \mathrm{HCl}_{100}$ & $0,5166^{0}$ \\
\hline $\mathrm{M}_{1} \operatorname{com} \mathrm{M}_{1 \mathrm{AT}}$ & $0,9146 * * *$ \\
\hline $\mathrm{M}_{1} \operatorname{com~} \mathrm{HCl}_{50 \mathrm{AT}}$ & $0,6498 *$ \\
\hline $\mathrm{M}_{1} \operatorname{com} \mathrm{HCl}_{100 \mathrm{AT}}$ & $0,7442 * *$ \\
\hline $\mathrm{HCl}_{50}$ com $\mathrm{HCl}_{100}$ & $0,9140 * * *$ \\
\hline $\mathrm{HCl}_{50}$ com $\mathrm{M}_{1 \mathrm{AT}}$ & $0,8227 * * *$ \\
\hline $\mathrm{HCl}_{50} \operatorname{com~} \mathrm{HCl}_{50 \mathrm{AT}}$ & $0,8896^{* * *}$ \\
\hline $\mathrm{HCl}_{50}$ com $\mathrm{HCl}_{100 \mathrm{AT}}$ & $0,8540 * *$ \\
\hline $\mathrm{HCl}_{100} \operatorname{com} \mathrm{M}_{1 \mathrm{AT}}$ & $0,7515 * *$ \\
\hline $\mathrm{HCl}_{100} \operatorname{com} \mathrm{HCl}_{50 \mathrm{AT}}$ & $0,7935 * *$ \\
\hline $\mathrm{HCl}_{100}$ com $\mathrm{HCl}_{100 \mathrm{AT}}$ & $0,8012 * *$ \\
\hline
\end{tabular}

${ }^{(1)} \mathrm{M}_{1}$ : Mehlich-1; $\mathrm{HCl}_{50}$ e $\mathrm{HCl}_{100}$ : $\mathrm{HCl} 50$ e $100 \mathrm{mmol} / \mathrm{L}$, respectivamente; AT: dosagem com adição do ácido tioglicólico. ${ }^{0}$, *, ** e ***Significativo a $10 \%, 5 \%, 1 \%$, e $0,1 \%$ de probabilidade, respectivamente, pelo teste $\mathrm{F}$.

Tabela 5. Coeficientes de correlação linear simples entre os teores de $\mathrm{B}$ extraídos com solução de $\mathrm{BaCl}_{2} 5 \mathrm{mmol} / \mathrm{L} \mathrm{e}$ aquecimento no forno de microondas, e os teores de $\mathrm{B}$ extraídos com Mehlich-1 e $\mathrm{HCl}$, sem adição (SAT) e com adição (CAT) de ácido tioglicólico, usando-se azometina-H para dosagem.

\begin{tabular}{lc}
\hline Variáveis & $\mathrm{BaCl}_{2} 5 \mathrm{mmol} / \mathrm{L}$ \\
\hline Mehlich-1, SAT & $0,3577^{\mathrm{ns}}$ \\
Mehlich-1, CAT & $0,3320^{\mathrm{ns}}$ \\
$\mathrm{HCl} 50 \mathrm{mmol} / \mathrm{L}, \mathrm{SAT}$ & $0,4879^{\mathrm{ns}}$ \\
$\mathrm{HCl} 50 \mathrm{mmol} / \mathrm{L}, \mathrm{CAT}$ & $0,2968^{\mathrm{ns}}$ \\
$\mathrm{HCl} 100 \mathrm{mmol} / \mathrm{L}, \mathrm{SAT}$ & $0,6181^{*}$ \\
$\mathrm{HCl} 100 \mathrm{mmol} / \mathrm{L}, \mathrm{CAT}$ & $0,3069^{\mathrm{ns}}$ \\
\hline
\end{tabular}

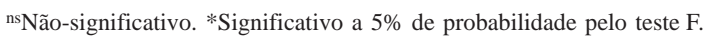


extrator na predição da ocorrência de deficiência de B na planta. Corre-se o risco de se escolher um extrator que na análise de um solo não adubado dê origem a teores de $\mathrm{B}$ que não correspondam aos valores reais de $\mathrm{B}$ no solo, teores esses que teriam pouca ou nenhuma relação com a absorção de B pelas plantas.

\section{Conclusões}

1. A presença de Fe em extratos de solo obtidos com Mehlich-1, $\mathrm{HCl} 50 \mathrm{mmol} / \mathrm{L} \mathrm{e} \mathrm{HCl} 100 \mathrm{mmol} / \mathrm{L}$ interfere na determinação do $\mathrm{B}$ disponível quando se faz a dosagem com azometina- $\mathrm{H}$.

2. Os teores de $\mathrm{B}$ determinados em extratos de solo obtidos com Mehlich-1, $\mathrm{HCl} 50 \mathrm{mmol} / \mathrm{L}$ e $\mathrm{HCl}$ $100 \mathrm{mmol} / \mathrm{L}$ não se correlacionam bem com os teores de $\mathrm{B}$ determinados em extratos obtidos com o $\mathrm{BaCl}_{2} \cdot 2 \mathrm{H}_{2} \mathrm{O} 5 \mathrm{mmol} / \mathrm{L}$, quando a dosagem é feita com azometina-H.

3. Na eliminação da interferência do Fe na determinação do B disponível em solos com características semelhantes às dos solos utilizados, não se recomenda a adição do ácido tioglicólico aos extratos obtidos com Mehlich-1 e HCl (50 mmol/Le $100 \mathrm{mmol} / \mathrm{L}$ ) para dosagem com azometina-H.

\section{Referências}

ABREU, C. A.; ABREU, M. F.; RAIJ, B. van; BATAGLIA, O. C.; ANDRADE, J. C. Extraction of boron from soil by microwave heating for ICP-AES determination. Communications in Soil Science and Plant Analysis, New York, v. 25, p. 3321-3333, 1994.

BATAGLIA, O. C.; RAIJ, B. van. Eficiência de extratores na determinação do boro em solos. Revista Brasileira de Ciência do Solo, Campinas, v. 14, p. 25-31, 1990.

BERGER, K. C.; TRUOG, K. Boron determination in soils and plants using the quinalizarin reaction. Industrial and Engineering Chemistry, Washington, v. 11, p. 540-545, 1939.

BINGHAM, F. T. Boron. In: PAGE, A. L. (Ed.). Methods of soil analysis. 2. ed. Madison: American Society of Agronomy/Soil Science Society of America , 1982. pt. 2, p. 431-447. (Agronomy, 9).

COX, F. R.; KAMPRATH, E. J. Micronutrient soil tests. In: MORTVEDT, J. J.; GIORDANO, P. M.; LINDSAY,
W. L. (Ed.). Micronutrients in agriculture. Madison: Soil Science Society of America, 1972. p. 289-317.

DEFELIPO, B. V.; RIBEIRO, A. C. Análises químicas do solo: metodologia. Viçosa, MG: Imprensa Universitária, 1981. 17 p. (Boletim de Extensão, 29).

EMBRAPA. Centro Nacional de Pesquisa de Solos (Rio de Janeiro, RJ). Manual de métodos de análises de solos. 2. ed. Rio de Janeiro, 1997. 212 p.

FERREIRA, G. B.; FONTES, R. L. F.; FONTES, M. P. F.; ALVAREZ VENEGAS, V. H. Influência de algumas características do solo nos teores de boro disponível. Revista Brasileira de Ciência do Solo, Viçosa, MG v. 25, n. 1, p. 93-103, 2001.

GUPTA, U. C. Boron nutrition of crops. Advances in Agronomy, San Diego, New York, v. 31, p. 273-307, 1979a.

GUPTA, U. C. Some factors affecting the determination of hot-water-soluble boron from podzol soils using azomethine-H. Canadian Journal of Soil Science, Ottawa, v. 59 , p. $241-247,1979$ b.

HOU, J.; EVANS, L. J.; SPIERS, G. A. Chemical fractionation of soil boron. I. Method development. Canadian Journal of Soil Science, Ottawa, v. 76, p. 485-491, 1996.

JEFFREY, L. E.; McCALLUM, L. E. Investigation of a hot $0,01 \mathrm{M} \mathrm{CaCl}_{2}$ soil boron extraction procedure followed by ICP-AES analysis. Communications in Soil Science and Plant Analysis, New York, v. 19, p. 663-673, 1988.

LIMA FILHO, O. F. Calibração de boro e zinco para o cafeeiro (Coffea arabica L. cv. Catuaí amarelo). 1991. 100 f. Dissertação (Mestrado em Energia Nuclear na Agricultura) - Centro de Energia Nuclear na Agricultura, Piracicaba.

OFFIAH, O. O.; AXLEY, J. H. Soil testing for boron on acid soils. In: GUPTA, U. C. (Ed.). Boron and its role in crop production. Boca Raton: CRC Press, 1993. p. 105123.

PARKER, D. R.; GARDNER, E. H. The determination of hot-water-soluble boron in some acid Oregon soils using a modified azomethine- $\mathrm{H}$ procedure. Communications in Soil Science and Plant Analysis, New York, v. 12, p. 1311-1322, 1981.

PONNAMPERUMA, F. N.; CAYTON, M. T.; LANTIN, R. S. Dilute hydrochloric acids as an extractant for available zinc, copper and boron in rice soils. Plant and Soil, The Hague, v. 61, p. 297-310, 1981. 
RAIJ, B. van; BATAGLIA, C. Análise química de solo. In: FERREIRA, M. E.; CRUZ, M. C. P. (Ed.). Micronutrientes na agricultura. Piracicaba: Potafos/ CNPq, 1991. p. 333-356.

RENAN, L.; GUPTA, U. C. Extraction of soil boron for predicting its availability to plants. Communications in Soil Science and Plant Analysis, New York, v. 22, p. 1002-1012, 1991.

RIBEIRO, A. C.; TUCUNANGO SARABIA, W. A. Avaliação de extratores para zinco e boro disponíveis em latossolos do Triângulo Mineiro. Revista Brasileira de Ciência do Solo, Campinas, v. 8, p. 85-89, 1984.

SHUMAN, L. M. Chemical forms of micronutrients in soil. In: MORTVEDT, J. J.; COX, F. R.; SHUMAN, L. M.; WELCH, R. M. (Ed.). Micronutrients in agriculture. 2. ed. Madison: Soil Science Society of America, 1991. p. 113-144. (SSSA Book Series, 4).
SIMS, J. T.; JOHNSON, G. V. Micronutrient soil tests. In: MORTVEDT, J. J.; COX, F. R.; SHUMAN, L. M.; WELCH, R. M. (Ed.). Micronutrients in agriculture. 2. ed. Madison: Soil Science Society of America, 1991. p. 427-476. (SSSA Book Series, 4).

VANDERLEY, J. C. Boro em materiais de três solos do Município de Lavras, Estado de Minas Gerais. 1984. 96 f. Dissertação (Mestrado em Solos e Nutrição de Plantas) - Escola Superior de Agricultura Luiz de Queiroz, Piracicaba.

WEAR, S. Boron. In: BLACK, E. A. (Ed.). Methods of soil analysis. Madison: American Society of Agronomy, 1965. p. 1059-1063.

ZARCINAS, B. A. Suppression of iron interference in the determination of boron using the azomethine- $\mathrm{H}$ procedure. Communications in Soil Science and Plant Analysis, New York, v. 26, p. 713-729, 1995. 